\title{
Interactions and non-commutativity in quantum Hall systems
}

\author{
Frederik G. Scholtt ${ }^{*}$ and Biswajit Chakraborty \\ Institute of Theoretical Physics, University of Stellenbosch, \\ Stellenbosch 7600, South Africa and \\ Satyendra Nath Bose National Centre for Basic Sciences, \\ Block-JD, Sector-III, Salt Lake, Kolkata - 700098, India. \\ Sunandan Gangopadhyay \\ Satyendra Nath Bose National Centre for Basic Sciences, \\ Block-JD, Sector-III, Salt Lake, Kolkata - 700098, India. \\ Jan Govaert:\$ \\ Institute of Nuclear Physics, Department of Physics, Catholic University of Louvain 2, \\ Chemin du Cyclotron, B-1348 Louvain-la-Neuve, Belgium.
}

(Dated: August 30, 2018)

\begin{abstract}
We discuss the role that interactions play in the non-commutative structure that arises when the relative coordinates of two interacting particles are projected onto the lowest Landau level. It is shown that the interactions in general renormalize the non-commutative parameter away from the non-interacting value $\frac{1}{B}$. The effective non-commutative parameter is in general also angular momentum dependent. An heuristic argument, based on the non-commutative coordinates, is given to find the filling fractions at incompressibilty, which are in general renormalized by the interactions, and the results are consistent with known results in the case of singular magnetic fields.
\end{abstract}

PACS numbers: $11.10 . \mathrm{Nx}$

\section{INTRODUCTION}

The quantum Hall system has recently attracted considerable attention from the point of view of non-commutative quantum mechanics and quantum field theory [1]-[9] as it is probably the simplest physical realization of a noncommutative spatial geometry. Some time ago Dunne, Jackiw and Trugenberger [10 already observed this noncommutativity by noting that in the limit $m \rightarrow 0$ the $y$-coordinate is effectively constrained to the momentum canonical conjugate to the $x$-coordinate. This result can also be obtained [1, 12] by keeping the mass fixed and taking the limit $B \rightarrow \infty$. An alternative point of view is to keep the magnetic field and mass finite, but to project the position coordinates onto the lowest (or higher) Landau level. These projected operators indeed satisfy the commutation relation $(\hbar=e=m=c=1) \underline{13}$

$$
\left[P_{0} x P_{0}, P_{0} y P_{0}\right]=\frac{1}{i B} P_{0}=\frac{1}{i B}
$$

where $P_{0}$ denotes the projector onto the lowest Landau level, which is also just the identity operator on the projected subspace, as reflected in the last step.

This result allows a simple heuristic understanding of quantum Hall fluids. Recall the elementary uncertainty relation (see e.g. [14]) for two non-commuting operators $A$ and $B$

$$
\begin{gathered}
(\Delta A)^{2}(\Delta B)^{2} \geq \frac{1}{4}\langle i[A, B]\rangle^{2}, \\
(\Delta A)^{2}=\left\langle A^{2}\right\rangle-\langle A\rangle^{2}, \quad(\Delta B)^{2}=\left\langle B^{2}\right\rangle-\langle B\rangle^{2},
\end{gathered}
$$

where $\langle\cdot\rangle$ denotes the normalized expectation value in some state. Using this we note that the non-commutativity of the coordinates implies a lower bound to the area a particle in the lowest Landau level occupies. This bound follows

\footnotetext{
*fgs@sun.ac.za

†biswajit@bose.res.in

${ }^{\ddagger}$ sunandan@bose.res.in

§Jan.Govaerts@fynu.ucl.ac.be
} 
easily from (21) to be $\Delta A=4\left|\Delta\left(P_{0} x P_{0}\right)\right|\left|\Delta\left(P_{0} y P_{0}\right)\right| \geq \frac{2}{B} \equiv \Delta A_{0}$. This implies that the number of states available in a Landau level is $M=\frac{A}{\Delta A_{0}}=\frac{A B}{2}$. The filling fraction is defined in the usual way as $\nu=\frac{N}{M}=\frac{2 N}{A B}$, with $N$ the number of electrons. For fermions it then follows that at maximum filling of $p$ Landau levels the particles must occupy the minimal allowed area, i.e., $N \Delta A_{0}=p A$ and $\nu=p$ with $p$ integer. As the next available states are in the higher Landau level, separated in energy by the cyclotron frequency, one expects that the quantum fluid will be incompressible at these values of the filling fraction.

In the literature mentioned above the effect that interactions between electrons might have on the non-commutativity was not investigated. In Ref. [3] an harmonic potential between two interacting particles was considered, but there the non-commutativity of the center of mass coordinates was investigated, which is again, effectively, a Landau problem. In particular the analysis of Ref. 13 has been done in the absence of any interactions between particles. Given the conjectured equivalence between a non-commutative $U(1)$ Chern-Simons theory [ $[,[6]$ and the composite fermion description for the fractional Hall effect, which is an effective non-interacting theory for the interacting quantum Hall system, one would like to understand the relationship between non-commutativity and interactions better. A similar picture arises in the much simpler setting of non-commutative quantum Hall systems where it seems as if the fractional quantum Hall effect, associated with an interacting quantum Hall system, can effectively be described by a non-interacting non-commutative quantum Hall system [9], again suggesting an interplay between non-commutativity and interactions. Indeed, keeping the philosophy of the composite fermion picture in mind, which replaces electrons interacting through a short ranged repulsive interaction by non-interacting composite fermions moving in a reduced magnetic field, one would expect that the interactions must modify the commutation relation (11) as the magnetic field is reduced. A similar conclusion was reached from a completely different point of view in a recent article of the present authors [15]. Here we want to investigate this question in more detail using the approach of Ref. [13].

To set the scene we consider two interacting particles with the same masses and charges moving in a plane with constant magnetic field perpendicular to the plane. In the symmetric gauge the Hamiltonian is given by $(\hbar=e=$ $m=c=1)$

$$
\begin{aligned}
H & =\frac{1}{2}\left(\vec{p}_{1}-\vec{A}\left(\vec{x}_{1}\right)\right)^{2}+\frac{1}{2}\left(\vec{p}_{2}-\vec{A}\left(\vec{x}_{2}\right)\right)^{2}+V\left(\left|\vec{x}_{1}-\vec{x}_{2}\right|\right), \\
A_{i}(\vec{y}) & =-\frac{B}{2} \epsilon_{i j} y^{j}, \quad B \geq 0 .
\end{aligned}
$$

Introducing relative and center of mass coordinates through

$$
\vec{R}=\frac{1}{2}\left(\vec{x}_{1}+\vec{x}_{2}\right), \quad \vec{r}=\vec{x}_{1}-\vec{x}_{2},
$$

the Hamiltonian reduces to

$$
\begin{aligned}
& H=\frac{1}{4}(\vec{P}-2 \vec{A}(\vec{R}))^{2}+\left(\vec{p}-\frac{1}{2} \vec{A}(\vec{r})\right)^{2}+V(|\vec{r}|), \\
& \vec{P}=\left(\vec{p}_{1}+\vec{p}_{2}\right), \quad \vec{p}=\frac{1}{2}\left(\vec{p}_{1}-\vec{p}_{2}\right) .
\end{aligned}
$$

This is of course two decoupled problems. The center of mass motion corresponds to that of a particle with mass $M=2$ and charge $q=2 e=2$ moving in a magnetic field $B$, while the relative motion is that of a particle with reduced mass $\mu=\frac{m}{2}=\frac{1}{2}$ and charge $q=\frac{e}{2}=\frac{1}{2}$ moving in a magnetic field $B$ and radial potential $V(|\vec{r}|)$. The cyclotron frequency for both problems is $B$. The center of mass motion can clearly be analysed as in Ref.[13]; projection onto the lowest Landau level will lead to non-commutative coordinates $\left[P_{0} X P_{0}, P_{0} Y P_{0}\right]=\frac{1}{2 i B}$. Our analysis here concerns the relative motion. This might seem problematic as the potential $V(|\vec{r}|)$ lifts the degeneracy of the Landau levels so that one can apparently no longer think of projection onto Landau levels, and particularly the lowest Landau level. Closer inspection of the argument in Ref. 13] reveals, however, that the degeneracy is not essential. Indeed, the only requirement is that the subspace on which is to be projected is infinite dimensional as the non-commutative coordinates can only be realized in this case. A natural generalization of the analysis in Ref. 13. would therefore be to identify a low energy infinite dimensional subspace on which to perform the projection. In the case of short range interactions $V(|\vec{r}|)$, for which the interaction energy scale is much less than the cyclotron frequency, which is the situation normally assumed, this can still be done. The reason is that the spectrum for the relative motion will clearly be close to that of the Landau problem for large values of the relative angular momentum as the particles are then well separated. For small values of the angular momentum the potential will have its main effect. However, if the interaction energy scale is much less than the cyclotron frequency, one will still have well separated bands of eigenstates with the cyclotron frequency the energy scale determining the separation between bands and the interaction energy scale the separation within bands. We can therefore identify an infinite dimensional low energy subspace as the lowest 
Landau level perturbed by the interaction and proceed to study the commutation relations of the relative coordinates projected onto this subspace.

This paper is organized in the following way. In section II we describe the general projection procedure onto the low energy subspace. In section III we apply this procedure to a number of exactly soluble interacting models to obtain insight into the underlying physics.

\section{GENERAL PROJECTION ON THE LOW ENERGY SECTOR}

We start by recalling a few basic facts about the Landau problem. A particle moving on a plane, subjected to a perpendicular constant magnetic field $B$, has a discrete set of energy eigenstates, known as Landau levels, and are labelled as $|n, \ell\rangle$, where $n$ and $\ell$ are integers labelling the various Landau levels $(n)$ and the degenerate angular momentum eigenstates with integer eigenvalues $\ell(\geq-n)$ within the same Landau level $n$.

We focus on the relative motion of the two particles described by the second part of the Hamiltonian (5),

$$
H=\left(\vec{p}-\frac{1}{2} \vec{A}(\vec{r})\right)^{2}+\tilde{V}(|\vec{r}|)
$$

From the rotational symmetry this problem can be solved as usual through the separation of variables and the wave functions have the generic form

$$
\psi_{n, \ell}(\vec{r})=\langle\vec{r} \mid n, \ell\rangle=R_{n, \ell}(r) e^{i \ell \phi},
$$

where $R_{n, \ell}$ solves the radial equation

$$
\left[-\frac{\partial^{2}}{\partial r^{2}}-\frac{1}{r} \frac{\partial}{\partial r}+\frac{\ell^{2}}{r^{2}}-\omega_{c} \ell+\frac{1}{4} \omega_{c}^{2} r^{2}+V(|\vec{r}|)\right] R_{n, \ell}=E_{n, \ell} R_{n, \ell},
$$

$n$ is the principle quantum number and $\omega_{c}=B / 2$ is half of the cyclotron frequency. Under the conditions discussed in section \the separated bands of eigenstates will be labelled by the principle quantum number, $n$, while the states within a band will be labelled by the angular mometum $\ell$. In particular we assume that the lowest energy states are described by $n=n_{0}$ (say) and $\ell=0,1,2 \ldots$ where we noted from (8) that a change in sign of the angular momentum will require an energy of the order of the cyclotron frequency, so that negative angular momenta will not occur in the low energy sector, i.e., we are restricting to the lowest Landau level, perturbed by interactions.

We can now construct the projection operator on the low energy sector as

$$
P_{0}=\sum_{\ell=0}^{\infty}\left|n_{0}, \ell\right\rangle\left\langle n_{0} \ell\right|
$$

We now compute the projected relative coordinates

$$
\begin{aligned}
& P_{0} x P_{0}=\sum_{l, l^{\prime}=0}^{\infty}\left\langle n_{0}, \ell^{\prime}|x| n_{0}, \ell\right\rangle\left|n_{0}, \ell^{\prime}\right\rangle\left\langle n_{0}, \ell\right|, \\
& P_{0} y P_{0}=\sum_{l, l^{\prime}=0}^{\infty}\left\langle n_{0}, \ell^{\prime}|y| n_{0}, \ell\right\rangle\left|n_{0}, \ell^{\prime}\right\rangle\left\langle n_{0}, \ell\right|,
\end{aligned}
$$

with

$$
\begin{aligned}
\left\langle n_{0}, \ell^{\prime}|x| n_{0}, \ell\right\rangle & =\Omega_{\ell^{\prime}, \ell}\left(\delta_{\ell^{\prime}, \ell+1}+\delta_{\ell^{\prime}, \ell-1}\right) \\
\left\langle n_{0}, \ell^{\prime}|y| n_{0}, \ell\right\rangle & =-i \Omega_{\ell^{\prime}, \ell}\left(\delta_{\ell^{\prime}, \ell+1}-\delta_{\ell^{\prime}, \ell-1}\right) \\
\Omega_{\ell^{\prime}, \ell} & =\pi \int_{0}^{\infty} d r r^{2} R_{n_{0}, \ell^{\prime}}^{*} R_{n_{0}, \ell} .
\end{aligned}
$$

The commutator of the relative coordinates then yields

$$
\left[P_{0} x P_{0}, P_{0} y P_{0}\right]=2 i \sum_{\ell=0}^{\ell=\infty}\left|\Omega_{\ell, \ell+1}\right|^{2}\left[\left|n_{0}, \ell+1\right\rangle\left\langle n_{0}, \ell+1|-| n_{0}, \ell\right\rangle\left\langle n_{0}, \ell\right|\right]
$$


We now simply have to compute the matrix elements $\Omega_{\ell^{\prime}, \ell}$ to determine the commutator. For some potentials this can be done analytically and exactly, but in most cases one has to resort to approximations. In this regard we note that since the potential has radial symmetry, it will not mix different angular momentum sectors. Within a particular angular momentum sector there is of course no degeneracy of the Landau states, so that one can safely apply perturbation theory to compute the radial wave-functions $R_{n_{0}, \ell}$ and therefore matrix elements $\Omega_{\ell^{\prime}, \ell}$. Indeed, this corresponds to a $1 / B$ expansion.

When the interaction is switched off $(V(r)=0)$ the radial wave-functions are those of the Landau problem and this result is easily seen to reduce to (1), except for a factor of two. The reason for this is simply that since we are working with the relative coordinates between two particles this commutator should yield in the non-interacting case the minimal area occupied by two particles, which is consistent with (1).

In contrast to the non-interacting case (1) this commutator is in general no longer proportional to $P_{0}$. However, since we are dealing with a central potential, the different angular momentum $(\ell)$ sectors decouple and one can interpret this result as a non-commutative theory with an effective $\ell$ dependent non-commutative parameter in the same spirit as was done in Ref. [15].

As the area occupied by the two particles will increase with increasing relative angular momentum, one can deduce from (12) and (2) an absolute lower bound to the average area that a particle in the low energy sector may occupy

$$
2 \Delta A=4\left|\Delta\left(P_{0} x P_{0}\right)\right|\left|\Delta\left(P_{0} y P_{0}\right)\right| \geq 4\left|\Omega_{0,1}\right|^{2} .
$$

The factor of two on the left is required as the right hand side is the average area occupied by two particles, as pointed out earlier.

\section{NON-COMMUTATIVITY IN SOME SOLUBLE MODELS}

In this section we study the non-commutative structure that arises in a number of soluble interacting models to gain deeper insight into the underlying physics.

\section{A. Harmonic oscillator}

We take for $V(|\vec{r}|)=\frac{\lambda^{2}}{4} r^{2}$. This is not a short range potential and the spectrum will not approach that of the Landau problem for large values of $\ell$. Indeed, here one gets a spectrum linearly growing in $\ell$ (see Fig 1 so that one cannot claim that projection onto the lowest principle quantum number will correspond to the lowest energy sector. However, as was pointed out in the introduction, one can in principle project onto any infinite dimensional subspace, not necessarily just the lowest energy, and that is the spirit in which the current calculation is done.

In this case it is easy to solve the radial equation for the lowest principle quantum number:

$$
\begin{aligned}
R_{0, \ell} & =N_{\ell} r^{\ell} \exp \left(-\frac{1}{4} \sqrt{\omega_{c}^{2}+\lambda^{2}} r^{2}\right) ; \quad \ell \geq 0, \\
N_{\ell} & =\frac{\left(\omega_{c}^{2}+\lambda^{2}\right)^{(\ell+1) / 2}}{\sqrt{\pi 2^{\ell+1} \Gamma(\ell+1)}} .
\end{aligned}
$$

The spectrum is given by

$$
E_{0, \ell}=\ell\left(\sqrt{\omega_{c}^{2}+\lambda^{2}}-\omega_{c}\right)+\sqrt{\omega_{c}^{2}+\lambda^{2}}
$$

and is linearly growing with increasing $\ell$. The spectrum and eigenfunctions for higher quantum numbers can of course also be solved easily and projection onto those subspaces can also be done. The full spectrum is given by $E_{n, \ell}=\sqrt{\omega_{c}^{2}+\lambda^{2}}(2 n+1)+\ell\left(\sqrt{\omega_{c}^{2}+\lambda^{2}}-\omega_{c}\right), n=0,1,2 \ldots, \ell \geq-n$ and is shown in Fig 1 for $\omega_{c}=1$ and $\lambda=0.5$. As no new features appear we restrict ourselves here to the solutions with the lowest principle quantum number.

The commutator of the relative coordinates can now be evaluated from (12) and yields

$$
\begin{aligned}
{\left[P_{0} x P_{0}, P_{0} y P_{0}\right] } & =\frac{i}{\sqrt{\omega_{c}^{2}+\lambda^{2}}} \sum_{\ell=0}^{\ell=\infty}(\ell+1)\left[\left|n_{0}, \ell+1\right\rangle\left\langle n_{0}, \ell+1|-| n_{0}, \ell\right\rangle\left\langle n_{0}, \ell\right|\right] \\
& =\frac{1}{i \sqrt{\omega_{c}^{2}+\lambda^{2}}} P_{0} \\
& =\frac{2}{i B}\left(1+\frac{4 \lambda^{2}}{B^{2}}\right)^{-1 / 2} .
\end{aligned}
$$




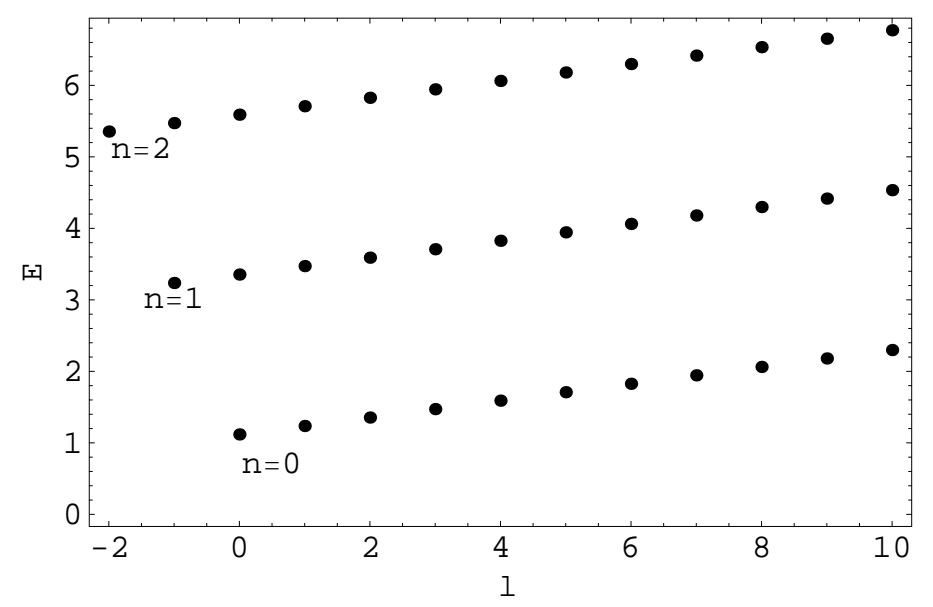

FIG. 1: The spectrum for the harmonic oscillator potential $\omega_{c}=1$ and $\lambda=0.5$.

In the last line we have noticed that $P_{0}$ is just the identity on the projected subspace. As was discussed in general, we note that when the interaction is switched off $(\lambda=0)$, this result differs by a factor of two from (11).

The first important point to note from this computation is that, generically, the non-commutative parameter is renormalized by the interactions.

We can follow the same heuristic line of reasoning as for the free case to compute the filling fractions at which the interacting quantum Hall fluid behaves incompressibly. The filling factor is $\nu=\frac{N}{M}=\frac{2 N}{A B}$. Arguing as in section [1] it follows from (17) that the average area occupied by a particle is strictly bounded from below by $2 \Delta A=$ $4\left|\Delta\left(P_{0} x P_{0}\right)\right|\left|\Delta\left(P_{0} y P_{0}\right)\right| \geq 4 / B \sqrt{1+\frac{4 \lambda^{2}}{B^{2}}} \equiv 2 \Delta A_{0}$. At maximum filling of the $p$ lowest Landau levels (bands) one expects the particles to occupy the minimum allowed area, i.e., $N \Delta A_{0}=p A$ and $\nu=p \sqrt{1+\frac{4 \lambda^{2}}{B^{2}}}, p$ integer. As the next available states are in the next Landau level, which are still separated on an energy scale of the cyclotron frequency under the assumption that the interaction energy scale is much less than the cyclotron frequency, one expects that the quantum fluid will be incompressible at these values of the filling. Note that these filling fractions are larger than the non-interacting values. This is easily understood from the attractive nature of the interaction which, effectively, enhances the magnetic field.

\section{B. Inverse square potential}

We take for $V(|\vec{r}|)=\frac{2 \lambda^{2}}{r^{2}}$. This Hamiltonian is very similar in structure to the Hamiltonian of a charged particle moving in a plane and coupled to the gauge potential $A_{i}=-\frac{\alpha}{r^{2}} \epsilon_{i j} x^{j}$ corresponding to a singular flux tube located at the origin, augmented by a harmonic potential. We investigate this case in detail in the next section as it is of particular importance in quantum Hall systems. Taking a cue from the wave function of this Hamiltonian [16], the lowest energy wave functions $(n=0, \ell \geq 0)$ are obtained by making the following ansatz:

$$
\psi_{n=0, \ell}(r, \phi)=N_{\ell} r^{\Lambda(\ell)} e^{i \ell \phi} \exp \left(-\frac{\omega_{c}}{4} r^{2}\right)
$$

where $\Lambda(\ell)$ is some unknown quantity which will get fixed by (8). The solution for $\Lambda(\ell)$, the exact low energy eigenvalues $E_{n=0, \ell}$ and the normalisation constant $N(\ell)$ are given by,

$$
\begin{gathered}
\Lambda(\ell)=\left(\ell^{2}+2 \lambda^{2}\right)^{1 / 2}, \\
E_{n=0, \ell}=\left[\left(\ell^{2}+2 \lambda^{2}\right)^{1 / 2}-\ell+1\right] \omega_{c}, \\
N_{\ell}=\left[\frac{\omega_{c}^{\Lambda(\ell)+1}}{\pi 2^{\Lambda(\ell)+1} \Gamma(\Lambda(\ell)+1)}\right]^{1 / 2} .
\end{gathered}
$$




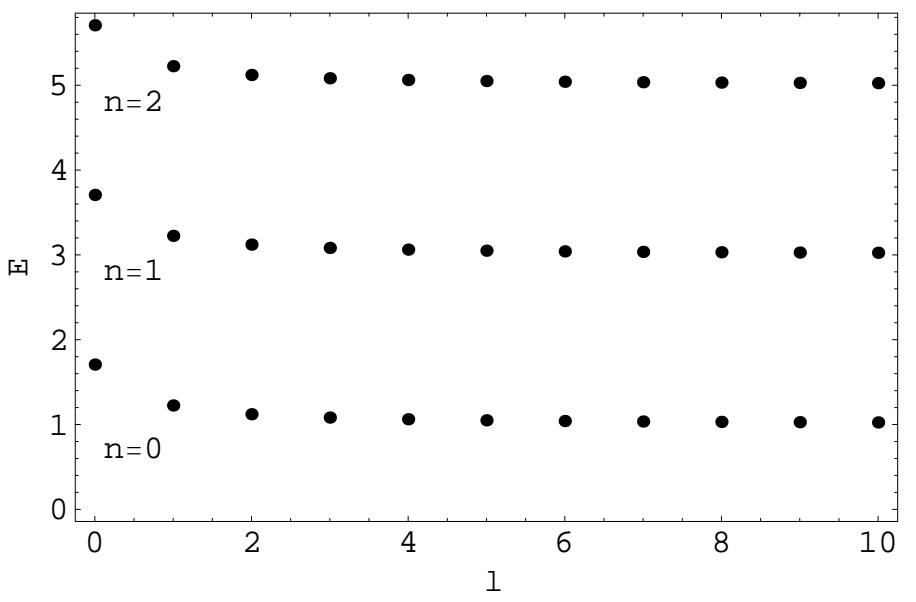

FIG. 2: The spectrum for the inverse square potential with $\omega_{c}=1$ and $\lambda=0.5$.

In this case the eigenvalues and eigenfunctions for higher Landau levels can also be solved as in Ref. 16]. The full spectrum is given by $E_{n, \ell}=\left[2 n+\left(\ell^{2}+2 \lambda^{2}\right)^{1 / 2}-\ell+1\right] \omega_{c}, n=0,1,2 \ldots, \ell \geq 0$ and is shown in Fig 2 for $\omega_{c}=1$ and $\lambda=0.5$. The expected features for a short range repulsive interaction can clearly be seen from this graph.

The commutator of the relative coordinates can now be evaluated from (12) and yields

$$
\left[P_{0} x P_{0}, P_{0} y P_{0}\right]=\frac{2 i}{B} \sum_{\ell=0}^{\infty} F(\ell)^{2}[|0, \ell+1\rangle\langle 0, \ell+1|-| 0, \ell\rangle\langle 0, \ell|]
$$

where $F(\ell)$ is given by

$$
F(\ell)=\frac{\Gamma\left(\frac{\Lambda(\ell)+\Lambda(\ell+1)+3}{2}\right)}{[\Gamma(\Lambda(\ell)+1) \Gamma(\Lambda(\ell+1)+1)]^{1 / 2}} .
$$

Note that in this case the right hand side of (22) is not proportional to the projection operator $P_{0}$ and, as pointed out earlier, one should interpret this as an effective non-commutative theory with an $\ell$-dependent renormalized noncommutative parameter.

Note that contrary to what one might naively expect, the lower bound of the area of the particle in angular momentum sector $l$, given in terms of the quantities $\left|F(\ell-1)^{2}-F(\ell)^{2}\right|$, are not monotonically increasing functions of $\ell$. To understand this, one must note that this lower bound is only achieved for minimum uncertainty states. The actual area is to be computed from $\left\langle r^{2}\right\rangle$ in the appropriate eigenstate, which is indeed a monotonically increasing function of $\ell$. One therefore concludes that the corresponding expression, evaluated at $\ell=0$ gives an absolute lower bound. Arguing as before, it follows from (22) that the average area occupied by a particle is strictly bounded from below by $2 \Delta A=4\left|\Delta\left(P_{0} x P_{0}\right)\right|\left|\Delta\left(P_{0} y P_{0}\right)\right| \geq \frac{4 F(0)^{2}}{B} \equiv 2 \Delta A_{0}$. As before the filling fractions at which the fluid is incompressible are $\nu=\frac{p}{F(0)^{2}}, p$ integer. As $F(0)^{2} \geq 1$ this yields a fractional filling factor.

\section{Singular magnetic fields}

In this section we consider the relative motion of the two particles without any interaction, but with a singular flux tube located at the position of the particles. To obtain the appropriate Hamiltonian [16], we perform a singular gauge transformation in the relative coordinate on the Hamiltonian (5). To be precise we perform the gauge transformation $e^{i \alpha \phi} H e^{-i \alpha \phi}$ with $\phi=\tan ^{-1}\left(\frac{y}{x}\right)$, with $y$ and $x$ the components of the relative coordinates. Dropping the center of mass part of (5), which is not affected by the gauge transformation, the gauge transformed Hamiltonian for the relative coordinate, which corresponds to a singular flux tube inserted at the position of the particles, reads [16]

$$
H=\left(\vec{p}-\frac{1}{2} \vec{A}\right)^{2},
$$


where

$$
A_{i}=-\left(\frac{B}{2}+\frac{2 \alpha}{r^{2}}\right) \epsilon_{i j} x^{j}, B \geq 0 .
$$

Written out explicitly the Hamiltonian reads

$$
H=-\frac{\partial^{2}}{\partial r^{2}}-\frac{1}{r} \frac{\partial}{\partial r}+\frac{1}{r^{2}}\left(i \frac{\partial}{\partial \phi}+\alpha\right)^{2}+i \omega_{c} \frac{\partial}{\partial \phi}+\frac{1}{4} \omega_{c}^{2} r^{2}+\alpha \omega_{c}
$$

As in the composite fermion paradigm, we choose $\alpha \leq 0$ so that it leads to an effective reduction of the magnetic flux seen by the particles. The low energy eigenfunctions and spectrum are easily found to be 16 .

$$
\begin{aligned}
\psi_{0, \ell} & =N_{\ell} r^{|\ell-\alpha|} e^{i \ell \phi} e^{-\frac{\omega_{c} r^{2}}{4}}, \ell \geq 0, \\
N_{\ell} & =\left[\frac{\omega_{c}^{|\ell-\alpha|+1}}{\pi 2^{|\ell-\alpha|+1} \Gamma(|\ell-\alpha|+1)}\right]^{1 / 2}, \\
E_{0, \ell} & =\omega_{c} .
\end{aligned}
$$

The commutator of the relative coordinates yields from (12)

$$
\begin{aligned}
{\left[P_{0} x P_{0}, P_{0} y P_{0}\right] } & =\frac{2 i}{B} \sum_{\ell=0}^{\infty}(l+|\alpha|+1)[|0, \ell+1\rangle\langle 0, \ell+1|-| 0, \ell\rangle\langle 0, \ell|] \\
& =\frac{2}{i B} \sum_{\ell=0}^{\infty}\left(|\alpha| \delta_{0, \ell}+1\right)|0, \ell\rangle\langle 0, \ell| .
\end{aligned}
$$

As before, it follows from (28) that the average area occupied by a particle is strictly bounded from below by $2 \Delta A=4\left|\Delta\left(P_{0} x P_{0}\right)\right|\left|\Delta\left(P_{0} y P_{0}\right)\right| \geq \frac{4(1+|\alpha|)}{B} \equiv 2 \Delta A_{0}$ and the filling fractions at which the fluid is incompressible are $\nu=\frac{p}{1+|\alpha|}, p$ integer. Keeping in mind the phase factor associated with the singular gauge transformation, unchanged statistics requires, as usual, that one must choose $\alpha=2 k$ with $k$ a negative integer. This choice indeed yields the fractional fillings as obtained from the composite fermion picture [17] when appropriate choices of $p$ and $k$ are made.

\section{DISCUSSION AND CONCLUSIONS}

We discussed the role that interactions play in the non-commutative structure that arises when the relative coordinates of two interacting particles are projected onto the lowest Landau level. It was shown that the interactions in general renormalize the non-commutative parameter away from the non-interacting value $\frac{1}{B}$. The effective noncommutative parameter is in general also angular momentum dependent, as was also found from other considerations in Ref. 15]. An heuristic argument, based on the non-commutative coordinates, was given to find the filling fractions at incompressibility and the results are consistent with known results in the case of singular magnetic fields. It should, however, be kept in mind that this argument was very simplistic as all possible many-body correlations were ignored. Probably due to this oversimplification this argument cannot explain, for a general short range repulsive interaction, the quantized values of the filling fraction at incompressibility observed in the fractional quantum Hall effect. Indeed, from naive perturbative considerations in the above setting one would expect that the (screened) Coulomb interaction will have only a perturbative effect on the non-commutative parameter and filling fraction, which is certainly not the case. As in other treatments, it is only when one already assumes the existence of composite fermions, as was done in section [IIC] that the quantized filling fraction can be explained. The apparently non-perturbative microscopic origin of composite fermions as effective non-interacting degrees of freedom to describe the Coulomb interacting quantum Hall fluid is, indeed, still an illusive and controversial issue [18].

\section{Acknowledgements}

This work was supported by a grant under the Indo-South African research agreement between the Department of Science and Technology, Government of India and the South African National Research Foundation. FGS would like to thank the S.N. Bose National Center for Basic Sciences and the Institute of Nuclear Physics, University of 
Louvain, for their hospitality in the periods that parts of this work were completed. BC would like to thank the Institute of Theoretical Physics, Stellenbosch University for their hospitality during the period when part of this work was completed.

[1] Y.S. Myung and H.W. Lee, Noncommutative spacetime and the fractional quantum Hall effect, arXiv:hep-th/9911031

[2] C. Duval and P. Horváthy, Phys. Lett. B 479, 284 (2000); J. Phys. A 34, 10097 (2001).

[3] D. Bigatti and L. Susskind, Phys. Rev. D62, 066004 (2000).

[4] V.P. Nair and A.P. Polychronakos, Phys. Lett. B505, 267 (2001).

[5] S. Hellerman and M. Van Raamsdonk, JHEP 0110, 039 (2001).

[6] L. Susskind, The quantum Hall fluid and non-commutative Chern-Simons theory, arXiv:hep-th/0101029

[7] A.P. Polychronakos, JHEP 0104, 011 (2001).

[8] P. Horváthy, Ann. Phys. 299, 128 (2002).

[9] O.F. Dayi and A. Jellal, J. Math. Phys. 43, 4592 (2002).

[10] G.V. Dunne, R. Jackiw and C.A. Trugenberger, Phys. Rev. D 41, 661 (1990); G.V. Dunne and R. Jackiw, Nucl. Phys. B (Proc. Suppl.) 33C, 114 (1993).

[11] Y.S. Myung and H.W. Lee, Noncommutative geometry and anyonic field theory in the magnetic field, arXiv:hep-th/9910083

[12] A. de Veigy and S. Ouvry, Nucl. Phys. B 388715 (1992).

[13] N. Macris and S. Ouvry, J. Phys. A 35, 4477 (2002).

[14] S. Gasiorowicz, Quantum Physics, John Wiley, New York, 1974.

[15] F.G. Scholtz, B. Chakraborty, S. Gangopadhyay and A. Ghosh Hazra, Phys. Rev. D 71, 085005 (2005).

[16] A. Comtet, S. Mashkevich and A. Ouvry, Phys. Rev. D 52, 2594 (1995).

[17] J.K. Jain, Phys. Rev. Lett. 63, 199 (1989).

[18] M.I. Dyakonov, in: Recent Trends in Theory of Physical Phenomena in High Magnetic Fields, I.D. Vagner et al. (eds.), pp. 75-88, Kluwer Academic Publishers (2003), arXiv: cond-mat 0209206 